\title{
Vacuum Rabi Splitting Using a Single Quantum Dot in a Photonic Crystal Slab Nanocavity
}

\author{
H. Gibbs 1 , G. Khitrova ${ }^{1}$, J. Hendrickson 1 , T. Yoshie ${ }^{2}$, A. Scherer ${ }^{2}$, O. Shchekin ${ }^{3}$, D. Deppe ${ }^{3}$ \\ ${ }^{1}$ University of Arizona, USA; ${ }^{2}$ Caltech, USA; ${ }^{3}$ University of Texas-Austin, USA \\ hyattgibbs@att.net
}

\begin{abstract}
We report the observation of vacuum-field Rabi splitting (true strong coupling) between a single InAs quantum dot and a single photon in the mode of a photonic crystal slab nanocavity.

Taking advantage of the fact that quantum dot transitions shift to longer wavelength with increased temperature much faster than a nanocavity mode, we have scanned a quantum dot resonance through the nanocavity resonance; see Fig. 1(upper). The two photoluminescence (PL) peaks repel each other instead of following the crossing of the dot and cavity resonances; this anti-crossing behavior, characteristic of strong coupling [1], is shown more clearly by plotting the peak positions on an expanded scale; see Fig. 1(lower).
\end{abstract}

A temperature scan over a narrower range around the minimum splitting is shown in Fig. 2(a). The parameters that give the best fit of our strong coupling data to an analytic expression are: splitting $2 \mathrm{~g}=41.2 \mathrm{GHz}$, cavity decay rate $\kappa=$ $42.3 \mathrm{GHz}$, and dot dephasing rate $\gamma=21.5 \mathrm{GHz}$; see Fig. 2(b). The condition for strong coupling, that the splitting exceed the linewidth of each peak, is satisfied: $2 \mathrm{~g}=41.2 \mathrm{GHz}>(\kappa+\gamma) / 2=$ $31.9 \mathrm{GHz}$.

Essential to this observation was the high Q of the nanocavity. The GaAs nanocavity slab is surrounded by air and contains a $2 \mathrm{D}$ photonic crystal structure based on the design of S. Noda's group with three missing holes in a line and with the end holes shifted out [2]. Q's over 20,000 were measured, but no dot was found strongly coupled to those nanocavities. The $\mathrm{Q}$ of the cavity mode used was measured at high power to be $\sim 13,300$ (linewidth $\sim 0.08 \mathrm{meV}$ ), but this dropped to about 6000 at the low power needed to see strong coupling and single quantum dot transitions. The volume of the cavity mode is computed to be about $\left(\lambda_{\text {cav }} / \mathrm{n}\right)^{3} \cong 0.04 \mu \mathrm{m}^{3}$, where $\lambda_{\text {cav }}$ is the cavity mode wavelength and $n$ is the refractive index.
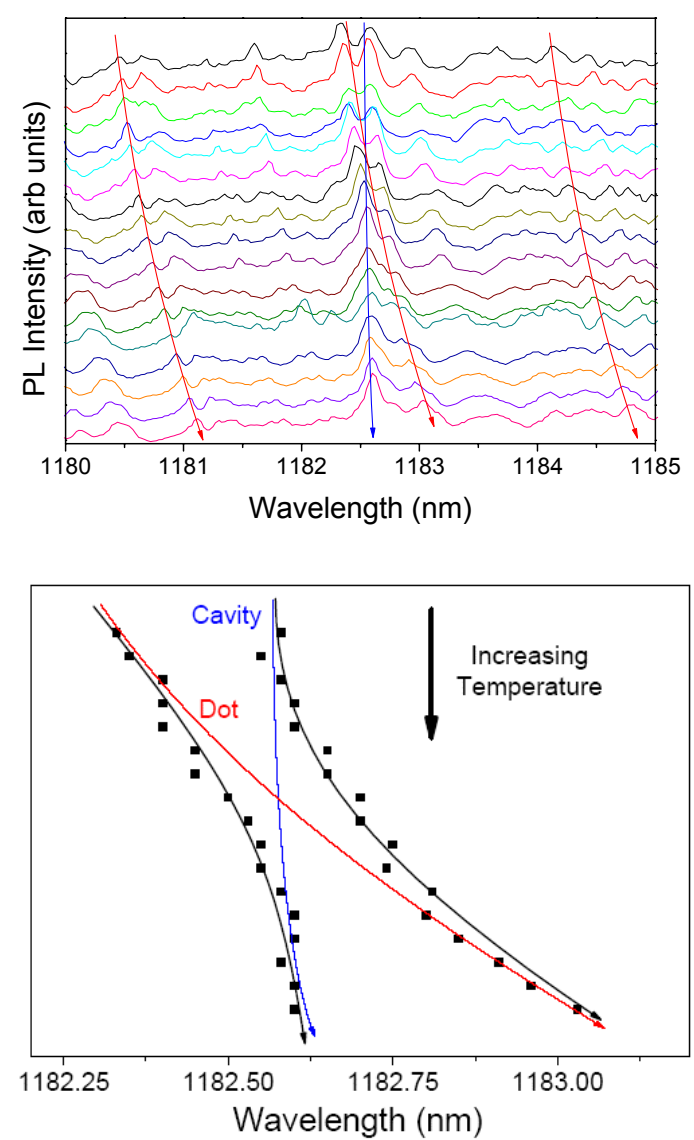

Fig. 1 Dot-nanocavity anti-crossing. (Upper) PL at low power $(0.78 \mu \mathrm{W})$ and 60 second average. The temperature is increased from $13 \mathrm{~K}$ at the top to $29 \mathrm{~K}$ at the bottom in $1 \mathrm{~K}$ steps. Note the anti-crossing around $20 \mathrm{~K}$. At the cavity wavelength, the background ensemble PL is as strong as the anti-crossing signal. The red curves show that all the quantum dot transitions shift the same with temperature and shift much faster than the cavity resonance given by the blue curve, taken from $25 \mu \mathrm{W}$ data where saturation of uncoupled dots makes the cavity peak dominate the PL spectrum. (Lower) The two coupled-system peaks [black lines are eye guides] are plotted as a function of temperature and compared with the scan rates of an uncoupled quantum dot [red curve] and the empty cavity [blue]. 


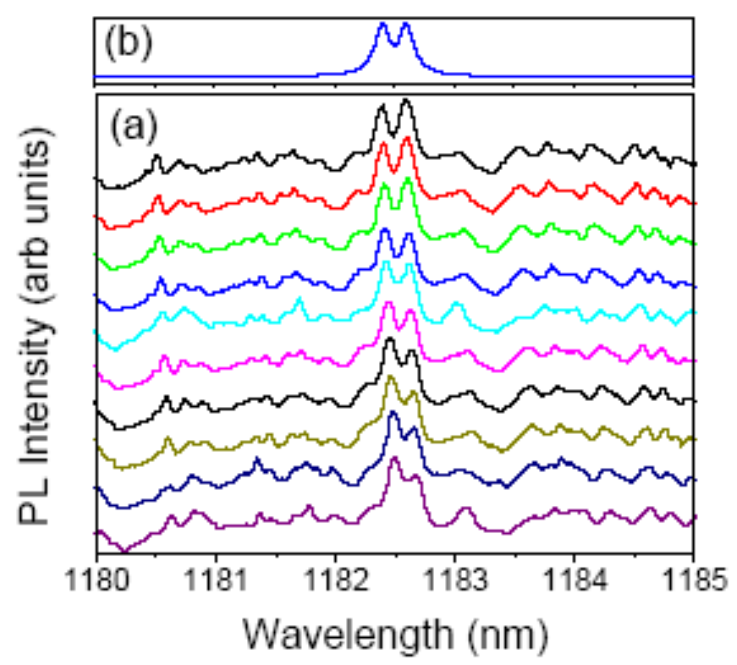

Fig. 2 Semiconductor strong coupling. The temperature is increased in $0.5 \mathrm{~K}$ steps from $15 \mathrm{~K}$ at the top to $19.5 \mathrm{~K}$ at the bottom, thereby changing the dot-nanocavity detuning in the vicinity of the minimum splitting and showing the double peaked photoluminescence characteristic of strong coupling. $780 \mathrm{nW}$ and 60 second average. (b) Plot of analytic expression for emission.

This breakthrough opens exciting possibilities for truly quantum optics cavity QED experiments in semiconductors and for solid-state entanglement needed for quantum information science.

The Tucson group thanks Eli Yablonovich for helpful suggestions and AFOSR, DURINT, and NSF AMOP and EPDT for support. The Caltech group thanks Dr. Susumu Noda and Dr. Yoshihiro Akahane for helpful discussions on the cavity designs, the MURI Center for Photonic Quantum Information Systems (ARO/ARDA), NSF ECS NIRT, and AFOSR. Finally, the Texas group acknowledges support from NSF ECS NIRT.

References

1) T. Yoshie, A. Scherer, J. Hendrickson, G. Khitrova, H. M. Gibbs, G. Rupper, C. Ell, O. B. Shchekin, and D. G. Deppe, Nature 432, 200 (2004).

2) Y. Akahane, T. Asano, B.-S. Song, and S. Noda, Nature 425, 944 (2003). 